\title{
sciendo
}

\section{A Systematic Review of Long-Distance Triathlon Musculoskeletal Injuries}

\author{
by \\ John-Henry Rhind ${ }^{1}$, Debashis Dass ${ }^{1}$, Andrew Barnett ${ }^{1}$, Michael Carmont ${ }^{2}$
}

The distribution of injuries affecting long-distance triathletes is yet to be fully understood. A systematic review was performed of the clinical literature to determine the epidemiology of musculoskeletal injuries affecting long-distance triathletes. Searched databases in Feb 2020 were PubMed, Medline, EMBASE, EMCARE, and CINHAL databases. Published observational research articles related to the incidence or prevalence of musculoskeletal injuries in longdistance triathletes (competing at "Ironman" full distance or greater), written in the English language and not restricted by age or gender or date were eligible. Of the 975 studies identified on the initial search, six studies met the inclusion criteria for analysis. The mean age (SD) of the long-distance triathletes in these studies was 35.1 (2.7) and the range was 21-68 years. Overuse injuries were most frequent with the incidence range of 37-91\%, and acute injury incidence range was $24-27 \%$. The knee and spine were the most frequent location of injury. Running and cycling were the most frequently affected disciplines. Elite athletes had a lower incidence of overuse injury (37\%). The highest acute injury incidence $(27 \%)$ was recorded in non-elite athletes. The quality of the studies was relatively poor with only one study satisfying $>50 \%$ of the quality assessment tool questions and only two studies were prospective, the rest were retrospective cross-sectional studies. Overall, there is a lack of literature reporting on musculoskeletal injuries in longdistance triathletes. Overuse injuries, particularly in the knee, are the most frequently reported, running and cycling are the most frequent disciplines associated. Long-distance triathletes may have a lower incidence of both overuse and acute injuries.

Key words: sports injury, ultra triathlon, endurance, ironman, running, cycling, swimming.

\section{Introduction}

The sport of Triathlon, consisting of a continuous successive swimming, cycling and running race, has progressed from a popular recreational event to an elite level sport being incorporated as a full Olympic sport in 2000. Triathlon has had an increasing number of participants as time has progressed. In the UK, British Triathlon membership has increased by over $200 \%$ since 2009 and race starts by $82 \%$ since 2009 (British Triathlon Statistics). The largest number of participants are in the shorter Sprint and Olympic distances, however, the most popular and prestigious long-distance triathlon event is called "Ironman" in which athletes race over a $3.9 \mathrm{~km}$ swim, a $180 \mathrm{~km}$ bicycle ride and a 42 $\mathrm{km}$ run (IRONMAN). This event is hugely popular as more than 90,000 athletes competed in IRONMAN events around the world in 2019. Within greater triathlon, the same distance is also known as a full or long- distance triathlon. Even more extreme triathlon events, such as Ultraman (Ultraman World Championships) or the Norseman race, also exist (A Journey like No Other - Xtri World Tour), but have fewer participants.

In common with many endurance sports there are challenges in determining injury rates with variations in injury definition and presentation, although some epidemiological data has been determined. Within the whole of triathlon, injury causing cessation of training for

1 - Robert Jones Agnes Hunt Hospital, Gobowen, Oswestry SY10 7AG, United Kingdom.

2 - Shrewsbury \& Telford Hospitals NHS Trust, United Kingdom. 
more than one day, reduction in training, or seeking medical aid could affect between 29 (Vleck, 2003) and 91\% O'Toole et al. (1989) of athletes. Overuse lower limb injuries are most frequently cited and running is the phase of sport most associated with injury (Caine et al., 2008; McHardy et al., 2006; Vleck et al., 2013).

Knowledge of event injury profiles is important for planning medical cover and useful in the return to activity, training and competition in these athletes. This may also aid future injury prevention in endurance training and racing in the future. To our knowledge, this is the first systematic review of long-distance triathlete musculoskeletal injuries at full distance or greater. Previous reviews have focused on triathlon as a whole, rather than long-distance specifically and are limited. It is not known how long-distance triathletes compare in terms of injury epidemiology with triathletes competing over shorter distances. Therefore, the aim of this study was to perform a systematic review to determine musculoskeletal injury epidemiology in long distance triathletes competing at full distance or greater.

\section{Methods}

The study was performed according to the Preferred Reporting Items for Systematic Reviews and Meta-Analysis (PRISMA) guidelines. The review was registered in the International Prospective Register of Systematic Reviews (PROSPERO) database (Registration number: CRD42020169743).

\section{Search Strategy}

Potentially relevant articles were identified using the NICE Healthcare Databases Advanced Search engine tool (HDAS, NHS Health Education England). This engine searches PubMed, Medline, EMBASE, EMCARE, and CINHAL databases. Search terms referred to keywords and where possible MeSH (Medical Subject Headings) terms were used. The search string was conducted as follows '(ironman* OR triathl*) AND (injur* OR back OR lumbar OR cervical OR thoracic OR shoulder* OR elbow* OR wrist* OR hand* OR hip* OR knee* OR ankle* OR foot $\mathrm{OR}$ feet $\mathrm{OR}$ leg OR arm OR Injury prevention)'. Results were filtered in English language, with all dates included up to the $14^{\text {th }}$ February, 2020, when the search was completed.
Bibliographic references of the papers were reviewed to ensure no relevant studies were missed. Attempts were made to contact the authors of articles when the data included was not clear. The search and strategy were performed by the authors and checked by a medical reference librarian.

\section{Selection Criteria}

Published epidemiological, observational research articles including case series, cohort studies, case-control or cross-sectional study designs were included. Articles were required to describe musculoskeletal injury incidence, prevalence, diagnosis, definition, localisation, severity, mechanism or treatment associated with long-distance triathletes. A long-distance triathlon was defined as a minimum of a $3.9 \mathrm{~km}$ swim, a $180 \mathrm{~km}$ bicycle ride and a $42 \mathrm{~km}$ run, the same as the Ironman with no upper distance limit of studies included. Articles were excluded when musculoskeletal injuries were not clearly attributable to a long-distance triathlon, either by training or competing. Due to competition rules of long-distance triathlons requiring athletes to be a minimum of 18 years old, inclusion was limited to adults of 18 years and above. Athletes of both sexes were included and of all experience levels: beginner, intermediate and elite. Articles were excluded based on the following criteria: case reports, editorials, commentaries, conference abstracts and opinion-based papers (Figure 1).

After the initial search, duplicates were removed. The remaining studies were selected for inclusion by title review according to the criteria. This excluded articles not related to triathlon. Articles were then reviewed by the abstract, followed by a full text review to ensure the study met the inclusion criteria. The review of the studies was conducted independently by two authors (JHR, DD). If either reviewer questioned inclusion, consensus was achieved following discussion. There was only one episode where consensus could not be met and a third reviewer (MC) was consulted.

\section{Risk of Bias}

All articles included for full text review were assessed using the Quality Assessment Tool for Observational Cohort and Cross-sectional studies framework (Appendix 1, Quality Assessment Tool for Observational Cohort and Cross-Sectional studies). This provided a 
framework for the authors to discuss and critically appraise the validity of a study. Studies were rated for risk of bias by two reviewers independently.

\section{Quality Assessment}

The overall quality of the studies included was considered to be poor and no single study answered all the questions (range $29-71 \%$, average 46.5\%; Appendix 1: Quality Assessment Tool for Observational Cohort and Cross-Sectional studies). The level of evidence was mainly level three, with one level two, these studies were predominantly retrospective cross sectional. Only two studies were prospective.

\section{Extraction and Data Analysis}

Data was extracted from the studies by two reviewers. Data extracted included: the level of expertise, the number of athletes included, age and sex, a response rate to the questionnaire and where possible, length of triathlete career. When analysing the types of injury, the following data was extracted: the study period, definition, diagnosis, mechanism, the type of acute injury, the type of overuse injury, anatomical site, severity, treatment and sub-discipline injury sustained in. Data analysis was performed in the form of reporting the variables and consolidating the data if comparable, such as the athletes' age. The level of statistical significance, where possible, was reported from each study. Means and standard deviations were calculated for demographical data using the statistical package R (3.4.3, R Core Team, Austria). A meta-analysis or further statistical analysis was not performed due to the heterogeneous nature of the data in each included paper.

\section{Results}

\section{Participant Demographics}

The total number of participants included in all studies was 5996. The mean (SD) age was 35.1 (2.67) years determined from four studies. Four studies reported on the sex of athletes, comprising 778 males and 158 females, $83 \%$ male and $17 \%$ female athletes. Age range (21-68 years old) was reported in one study (Egermann et al., 2003). The mean (SD) career length in Ironman athletes was 4.9 (1.6) years, this was calculated from three studies which reported the mean length of their overall triathlon career (Andersen et al., 2013; O'Toole et al., 1989; Vleck et al., 2010).
The mean (SD) age of elite and professional athletes was 33.7 (3.3) calculated from two studies (O'Toole et al., 1989; Vleck et al., 2010). One study reported sex of elite athletes in both male and female competitors, comprising of $75(79 \%)$ men and $20(21 \%)$ women (O'Toole et al., 1989). Mean length of career in elite Ironman athletes was 3.4 years, and it was reported in one study O'Toole et al. (1989).

The other studies included amateur athletes or a mixture of both amateur and professional athletes due to the small numbers of athletes competing in each of these events. The study locations were global and included locations in Europe and North America. Two of the six studies were standard Ironman events, two were Ironman world championship events in Hawaii (Hiller et al., 1987; O'Toole et al., 1989) and another an extreme Ironman event known as a Norseman Andersen et al. (2013).

\section{Data Collection}

Four of the studies used questionnaires (Andersen et al., 2013; Egermann et al., 2003; O'Toole et al., 1989; Vleck et al., 2010). These were retrospective cross-sectional studies ranging from one month following a specific long-distance triathlon event (Egermann et al., 2003; O'Toole et al., 1989) to 5 years retrospectively (Vleck et al., 2010). Andersen et al. (2013) collected data prospectively over a 26-week period, in which every two weeks participants completed an online questionnaire regarding injuries. Rimmer and Coniglione (2012) collected data from the medical tent where attendees received assessment and treatment of their injuries during the event. Hiller et al. (1987) retrospectively analysed medical records from three distances: United States Triathlon Series (Olympic distance), Ironman Qualifier (IQ) race (half Ironman) and Ironman World Championships. Only results attributable to long-distance triathlon events were included. The number of admissions were analysed from 1982 to 1985, and individual diagnoses were detailed for the 1984 and 1985 Ironman events, which were included.

The level of statistical significance was only reported in two of the studies (0.05) (Egermann et al., 2003; Vleck et al., 2010).

\section{Injury Incidence \& Prevalence}

An overview of the types of injury described in each study is shown in Table 2. The 
reporting of injuries varied based upon the period of investigation: single event, year or career. Two of the studies reported the incidence of overuse injuries over one year retrospectively (Hiller et al., 1987; Vleck et al., 2010). The range of overuse incidence was from 37 (Vleck et al., 2010) to 91\% (O'Toole et al., 1989). Egermann et al. (2003) reported an overall injury incidence of $75 \%$ with at least one injury over a 6.7-year Ironman career. Of these injured respondents, $76 \%$ had chronic complaints at one or more body regions. Andersen et al. (2013) calculated an average prevalence of overuse injuries (56\%), prospectively collected weekly over a 26-week period. The incidence and prevalence of acute injuries were less clearly reported. Andersen et al. (2013) reported $41(24 \%)$ acute injuries, out of 174 respondents. Rimmer and Coniglione (2012) reported $37 \%$ of non-elite Ironman athletes presented for medical attention. From the reported data the acute injury incidence was determined to be $27 \%$ after medical problems were excluded (Rimmer and Coniglione, 2012).

Two studies reported the incidence/1000 $\mathrm{hr}$ exposure to training or competition (Andersen et al., 2013; Egermann et al., 2003). Egermann et al. (2003) reported an overall injury incidence/1000 hr exposure to training of 0.71 . Andersen et al. (2013) reported the acute injury incidence/1000 hr exposure of 0.97 to training and 1.02 to competition.

Definition of injury, Localisation of injury, Severity of injury, Time to return to sport Definition

All of the included studies contained a definition of injury, however, these varied greatly and many studies did not explain clearly how overuse and acute injuries were differentiated. Two studies defined injury as a pain that led to the cessation of training or racing activities for at least a day (Egermann et al., 2003; Vleck et al., 2010). Alternatively, two studies defined injury as any presentation to the medical tent during an event (Hiller et al., 1987; Rimmer and Coniglione, 2012).

Only two studies defined both acute and overuse injuries (Andersen et al., 2013; Vleck et al., 2010). Andersen et al. (2013) used the Oslo Sports Trauma Research Centre (OSTRC) Overuse Injury Questionnaire to standardise reporting and was the only study to use this Clarsen et al. (2013).
Vleck et al. (2010) and Hiller et al. (1987) reported on injuries termed "orthopaedic" problems in Ironman athletes. In the absence of further definitions or data in their respective papers, these injuries were categorised as other injuries. O'Toole et al. (1989) left the definition of injury up to the triathlete to decide and instead relied upon athletes reporting the anatomical locations of perceived injury.

Localisation of Injury

Four of the studies detailed the patterns of overuse injuries by their anatomical location (Andersen et al., 2013; Egermann et al., 2003; O'Toole et al., 1989; Vleck et al., 2010). Each study varied in boundaries of the anatomical area. Rimmer and Coniglione (2012) gave the diagnoses, but not the anatomical locations of the injuries.

Hiller et al. (1987) did not include the location of injuries and instead focused primarily on the medical and physiological considerations of Ironman athletes. An overview of the anatomical locations of overuse injuries can be seen in Table 3.

Severity

Studies which measured severity varied in their definitions. Egermann et al. (2003) defined severity of injury by the level of medical treatment required: no treatment, low intensive medical treatment and those requiring hospitalisation. Vleck et al. (2010) used an injury severity index (the total time taken off training as the result of injury, by the athletes in either the Ironman (IM) or Olympic distance (OD) groups, divided by the number of athletes in that group) which was also compiled for each anatomical location. Rimmer and Coniglione (2012) categorised severity in athletes admitted to the medical tent. Patients were categorized as severe if they were lying down on a stretcher on arrival and were subsequently placed head down to reduce vascular pooling. Attendees were considered to be non-severe if they were able to sit up without assistance. Pure musculoskeletal categorisation was not performed. Andersen et al. (2013) used the severity score from the OSTRC Overuse Injury Questionnaire of 0-100 based upon the anatomical areas: shoulder, lower back, thigh, knee and lower leg (Clarsen et al., 2013).

Time to return to sport

Rimmer and Coniglione (2012) reported 
the time spent by athletes in the medical tent during an Ironman event. The average duration of emergency treatment increased to a peak of 62.3 min in hour 14 before reducing to a moderate level of $38.5 \mathrm{~min}$ and $33.7 \mathrm{~min}$ in hours 15 and 16, respectively. Andersen et al. (2013) detailed the time to return to training for overuse and acute injuries according to each anatomical area affected. Hip and foot/ankle injuries accounted for the longest return to training in overuse injuries (>28 days). The reporting of key variables used to describe injuries in long-distance triathletes in each study can be seen in

\section{Diagnosis, Risk factors, Mechanism, Treatment Diagnosis}

Four of the studies contained any diagnoses of musculoskeletal injuries (Andersen et al., 2013; Egermann et al., 2003; O'Toole et al.,1989; Rimmer and Coniglione 2012). Three of these detailed acute injury diagnoses (Andersen et al., 2013; Egermann et al., 2003; Rimmer and Coniglione, 2012). Whereas O'Toole et al. (1989) described diagnoses associated with overuse.

Two studies involved qualified clinicians diagnosing Ironman competitor's injuries compared with athletes' self-reporting (Hiller et al., 1987; Rimmer and Coniglione, 2012). Medical illnesses and physiological considerations such as dehydration were included in four studies as part of their analysis, in addition to musculoskeletal injuries (Andersen et al., 2013; Hiller et al., 1987; O'Toole et al., 1989; Vleck et al., 2010).

Risk Factors

Egermann et al. (2003), O'Toole et al. (1989) and Vleck et al. (2010) quantified risk according to each discipline: swimming, cycling and running. Egermann et al. (2003) identified a positive correlation between injury incidence and weekly training duration and competitions were associated with a six times injury incidence compared with training. Younger triathletes were associated with more contusions and abrasions whilst older athletes had more fractures Egermann et al. (2003). In athletes over 40 years old, $7.4 \%$ sustained fractures, whilst in those below the age of 30 , only $0.8 \%$ sustained fractures Egermann et al. (2003). Vleck et al. (2010) described previous injury as the greatest risk factor for further injury, and it was more likely to occur in running compared with cycling and swimming. The association of swimming, cycling and running to injury can be seen in Figure 2. Treatment

Three studies discussed treatment in any form (Egermann et al., 2003; Hiller et al., 1987; Rimmer and Coniglione, 2012). Egermann et al. (2003) observed that in $51.2 \%$ of all injuries no specific treatment was applied, low intensive medical treatment was needed in $27.6 \%$ and $21.2 \%$ of all injuries were treated by hospital or high intensive medical treatment. In studies reporting medical attendance, Hiller et al. (1987) calculated from four Ironman World Championship events (1982-1985) that $37 \%$ of athletes required intravenous (IV) fluid, on average 1.751 per athlete. Additionally, $0.3 \%$ were hospitalised and discharged (Hiller et al., 1987). Rimmer and Coniglione (2012) reported the first line treatment in those with exhaustion with rehydration and adopting a supine or a Trendelenburg position. Only $36 \%$ of the medical tent attendees required IV fluid. Neither study reported treatment methods for musculoskeletal injuries.

\section{Elite/Professional Ironman Athlete Injuries}

Five studies reported including elite or professional long-distance triathletes (Andersen et al., 2013; Hiller et al., 1987; O'Toole et al., 1989; Rimmer and Coniglione, 2012; Vleck et al., 2010). In most studies there was no differentiation of the level of performance and the injury data, although the Ironman is a unique event in which amateurs race with professionals. The study of Vleck et al. (2010) was the only one to include only elite or professional athletes, who finished within the top fifty of their respective national Olympic distance or full distance championships within the GB national squad. The rate of overuse injuries in this group was significantly lower than in the other non-elite studies. However, only a small sample of 19 athletes was studied. O'Toole et al. (1989) included 95 participants in their study (9\% of the race entrants) from the 1986 Ironman World Championships, $15 \%$ of athletes were professional and almost $50 \%$ were from professional occupations. Hiller et al. (1987) reported on medical admission from the 1982-1985 Ironman World Championships (4583 participants in total), but did not elaborate on how many of those athletes were elite or professional. The study of Rimmer and Coniglione (2012) was the only one to clearly report no elite competitors in their cohort and was therefore amateur. The 
presentation rate of $37 \%$ to the medical tent was

higher in non-elite Ironman athletes compared to elite ones.

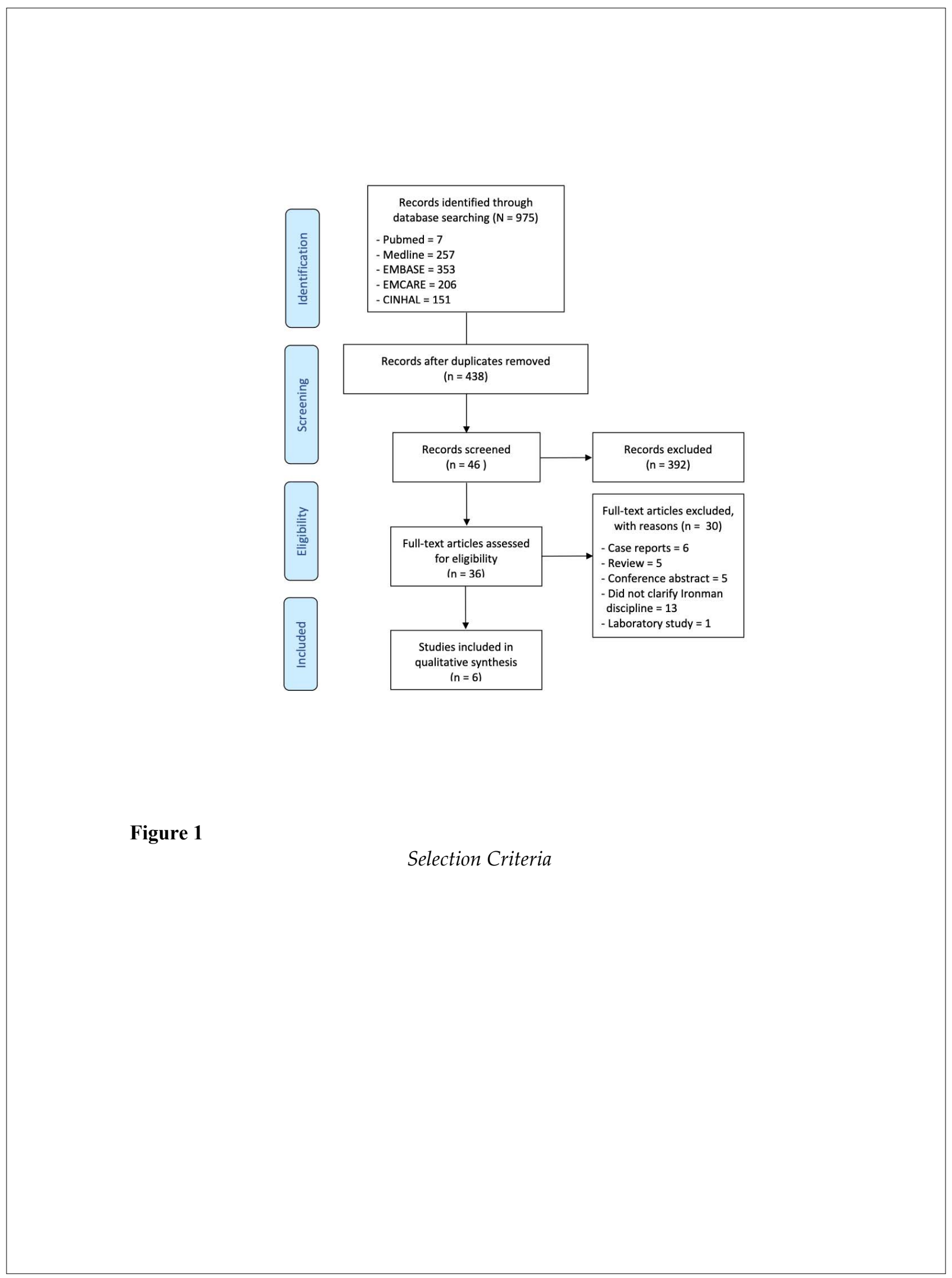




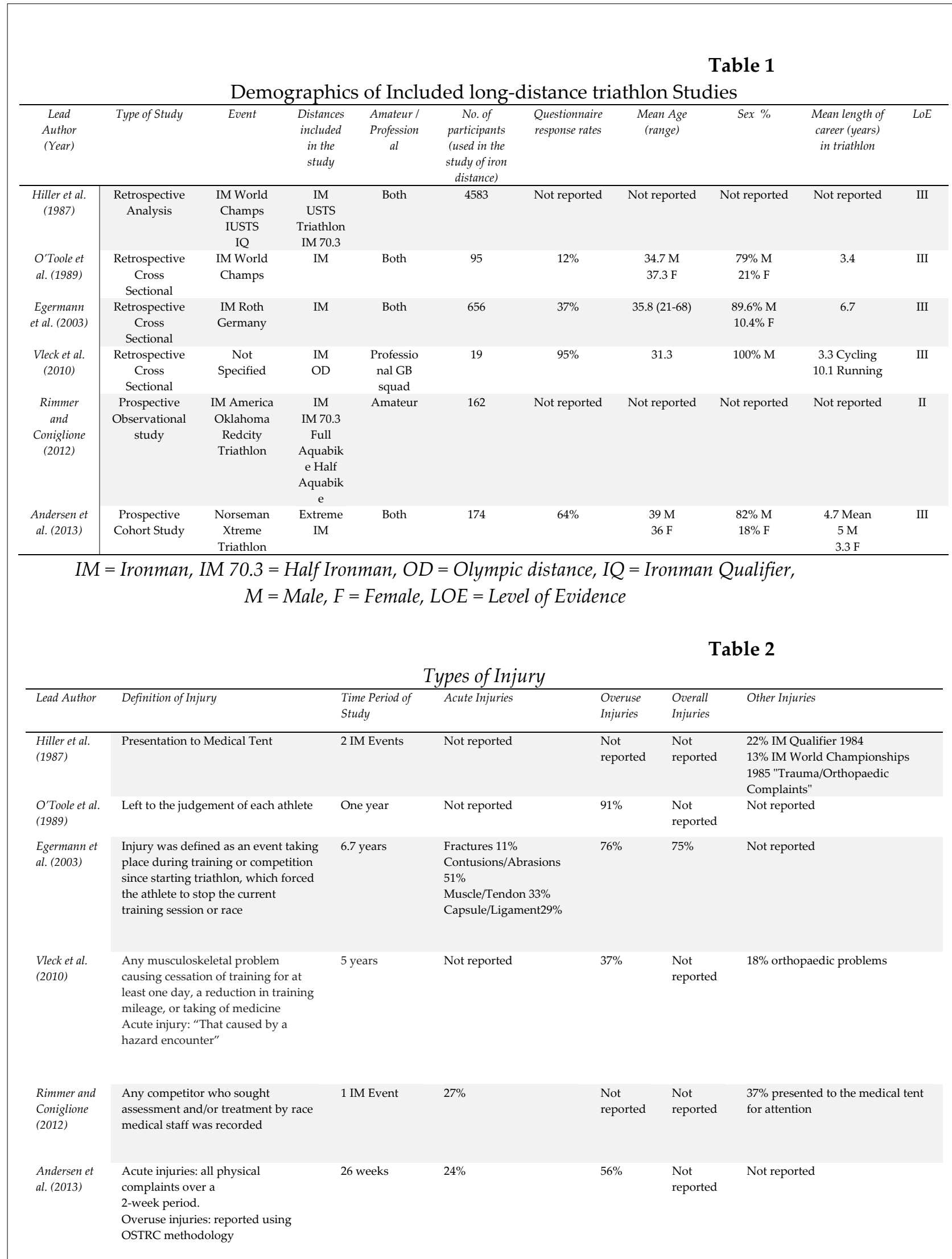

$I M=$ Ironman, IM 70.3 = Half Ironman, $O D=$ Olympic distance, $I Q=$ Ironman Qualifier 
Table 3

Overuse Injuries by Anatomical Location in long-distance triathletes

\begin{tabular}{|c|c|c|c|c|c|c|c|c|c|c|c|}
\hline Lead Author & Shoulder & Elbow & Back $^{*}$ & Ant Thigh & $\begin{array}{l}\text { Hamstring } \\
s\end{array}$ & Knee & Calf & Ankle/Foot & $\begin{array}{l}\text { Achilles } \\
\text { Tendon }\end{array}$ & Other & $>1$ injury \\
\hline $\begin{array}{l}\text { O'Toole et al. } \\
\text { (1989) }\end{array}$ & $\begin{array}{l}\text { Not } \\
\text { reported }\end{array}$ & $\mathrm{Na}$ & $72 \%$ & $63 \%^{*}$ & $\begin{array}{l}\text { Not } \\
\text { reported }\end{array}$ & $63 \%^{*}$ & $\begin{array}{l}\text { Not } \\
\text { reported }\end{array}$ & Not reported & $\begin{array}{l}\text { Not } \\
\text { reported }\end{array}$ & $\begin{array}{l}\text { Not } \\
\text { reported }\end{array}$ & $79 \%$ \\
\hline $\begin{array}{l}\text { Egermann et al. } \\
\text { (2003) }\end{array}$ & $19 \%$ & $4 \%$ & $31 \%$ & Not reported & $\begin{array}{l}\text { Not } \\
\text { reported }\end{array}$ & $43 \%$ & $\begin{array}{l}\text { Not } \\
\text { reported }\end{array}$ & $22 \%$ & $27 \%$ & $\begin{array}{l}\text { Not } \\
\text { reported }\end{array}$ & $\begin{array}{l}\text { Not } \\
\text { reported }\end{array}$ \\
\hline $\begin{array}{l}\text { Vleck et al. } \\
\text { (2010) }\end{array}$ & $8 \%$ & $\mathrm{Na}$ & $\begin{array}{l}29 \% \\
\text { Overall * }\end{array}$ & $8 \%$ & $20 \%$ & $44 \%$ & $20 \%$ & $0 \%$ & $12 \%$ & $8 \%$ & $\begin{array}{l}\text { Not } \\
\text { reported }\end{array}$ \\
\hline $\begin{array}{l}\text { Andersen et al. } \\
\text { (2013) }\end{array}$ & $42 \%$ & $\mathrm{Na}$ & $\begin{array}{l}46 \% \\
\text { Lower } \\
\text { Back } \\
\end{array}$ & $34 \%$ & $\begin{array}{l}\text { Not } \\
\text { reported }\end{array}$ & $44 \%$ & $\begin{array}{l}\text { Not } \\
\text { reported }\end{array}$ & Not reported & $\begin{array}{l}\text { Not } \\
\text { reported }\end{array}$ & $\begin{array}{l}\text { Not } \\
\text { reported }\end{array}$ & $\begin{array}{l}\text { Not } \\
\text { reported }\end{array}$ \\
\hline
\end{tabular}

* O'Toole et al. (1989) - 63\% kneelanterior thigh could not differentiate results further

* Back: Vleck et al. (2010) Andersen et al. (2013) categorised back into upper/lower lower back pain

* Vleck et al. (2010) further subdivided results: 20\% lower back, 9\% upper back

\section{Table 4}

A Heatmap Representing Injury Variables From Each Study
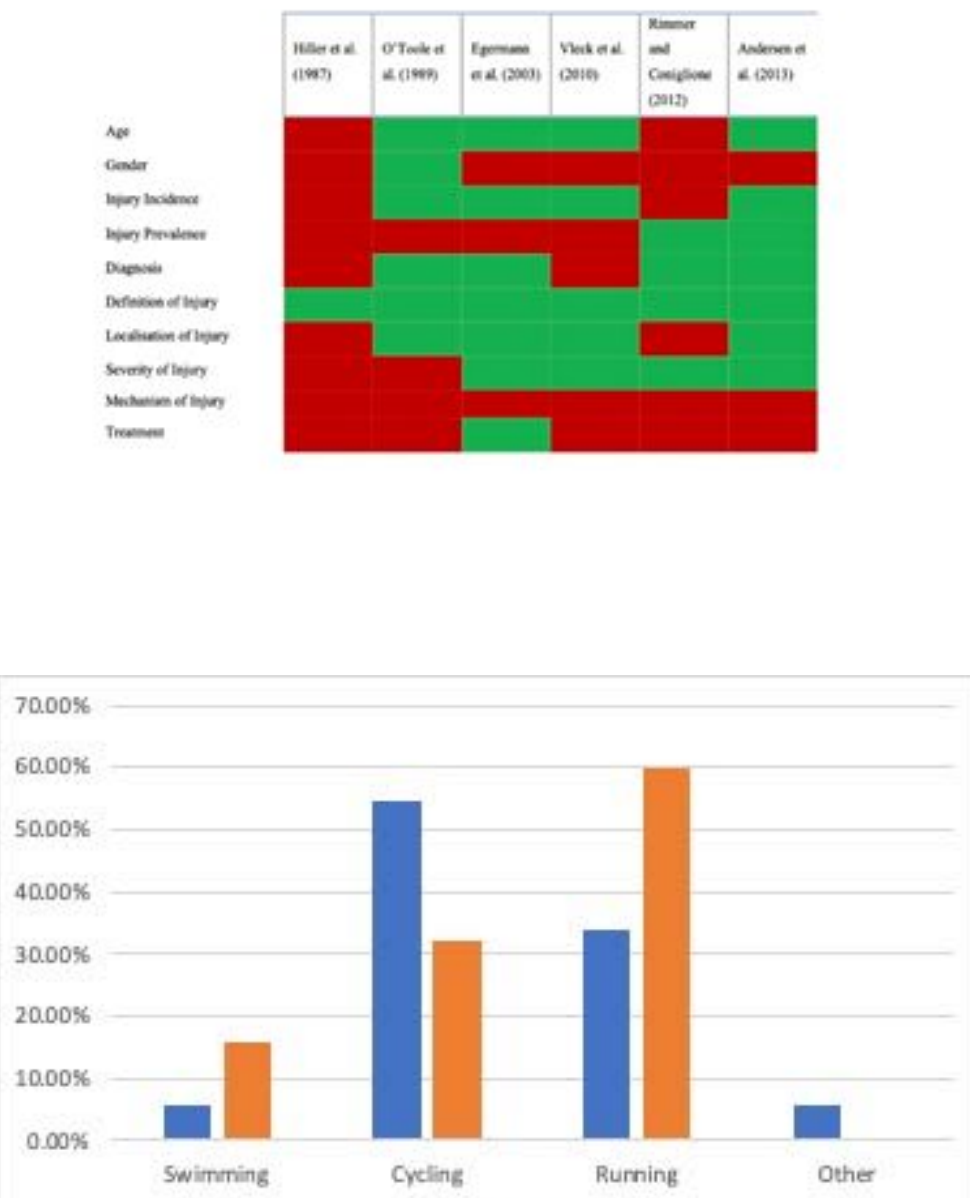

- Egermann et al. $(2003)$ Acute Injuries $\{11\}$. Vledk et al. (2010) overuse Injuries $\{12\}$

Figure 2

Percentage of injuries associated with: swimming, cycling $\mathcal{E}$ running 


\section{Discussion}

This is the first study to systematically review the current literature reporting the epidemiology of musculoskeletal injuries in longdistance triathletes. The principal finding is that there is a limited number of studies reporting the epidemiology of musculoskeletal injuries in longdistance triathlon and there is a lack of knowledge and consistency in this field. Inconsistent reporting of definitions and the general heterogeneity between studies made detailed comparison between studies, including statistical pooled analysis, not possible.

Overall, overuse injuries were the most frequently reported triathlon musculoskeletal injuries across all levels of participants. The incidence of overuse injuries in long-distance triathletes ranged from 37 to $91 \%$ (Andersen et al., 2013; Egermann et al., 2003; O'Toole et al., 1989; Vleck et al., 2010). This is similar to the range of overuse injuries (49 to $85 \%$ ) described in shorter distance triathlon triathletes in other studies (Burns et al., 2003; Collins et al., 1989; Korkia et al., 1994; Massimino et al., 1988; Vleck and Garbutt 1998; Wilk et al., 1995).

Overuse injury incidence in elite longdistance triathletes was also similar, whereas in the non-elite, the incidence was higher ranging from 56 to 76\% (Andersen et al., 2013; Egermann et al., 2003). Egermann et al. (2003) also reported an overall injury incidence/1000 $\mathrm{hr}$ exposure to training (in contrast to other studies) of 0.71 of which the majority related to overuse. Out of the six studies, Vleck et al. (2010) was the only direct comparison of the incidence of overuse injury in long-distance triathletes (37\%) compared with Olympic distance triathletes $(17 \%)$. The greater distances involving training and racing may predispose athletes to more overuse injuries.

Acute injuries in long-distance triathlon, in this study, ranged from 24 to $27 \%$ (Andersen et al., 2013; Rimmer and Coniglione, 2012); whereas acute injuries from other studies in shorter distance triathlon literature range from 15 to $56 \%$ (Massimino et al., 1988; Vleck et al., 2010). Longdistance triathletes tend to be more experienced and the fact that these athletes compete at slower speeds, particularly cycling with less pack riding, may reduce the acute injury incidence. The highest acute injury incidence (27\%) (Rimmer and Coniglione, 2012) was from non-elite athletes and the lowest overuse injury incidence (37\%) (Vleck et al., 2010) was from a cohort of elite team GB national squad long-distance triathletes, suggestive that experience is important and elite athletes may be less predisposed to injury. Andersen et al. (2013) included an acute injury incidence of $0.97 / 1000 \mathrm{hrs}$ training. In terms of acute injuries, there was no difference between short and long-distance triathletes surveyed, however, only a small number of longer distance athletes responded.

Egermann et al. (2003) showed a 6-fold increase in all injuries in competition compared to training amongst long-distance triathletes and Andersen et al. (2013) also reported an increased acute injury incidence/1000 $\mathrm{hr}$ competition. On average, long-distance triathletes compete less frequently than their shorter distance counterparts which may also affect overall injury rates. Many athletes reporting injuries sustained more than one, with an average of 2.9 injuries per athlete per year (O'Toole et al., 1989).

In this review, the lower extremity and in particular the knee, was the most common anatomical location affected by injury (Egermann et al., 2003; Vleck et al., 2010). This is similar to the literature for the whole of triathlon (Collins et al., 1989; Gosling et al., 2013; Ireland and Micheli, 1987; Kienstra et al., 2017; Korkia et al., 1994; Vleck and Garbutt, 1998; Shaw et al., 2004). Running is often recognised as the predominant risk factor for knee injuries in triathletes (Clements et al., 1999) and (Vleck et al. 2010) attributed most knee injuries to running. Diagnoses cited for knee pain in this review were chondromalacia patella, iliotibial band syndrome and tendinitis of the knee (O'Toole et al., 1989).

Back pain, particularly lumbar back pain, was the second most frequent injury location in this review, although in two of the studies back pain had the highest incidence (Andersen et al., 2013; O'Toole et al., 1989). There was, however, wide variation in definitions of back pain and some studies included the entire spine or divided into upper and lower back pain (Andersen et al., 2013; Vleck et al., 2010) together with sciatica (O'Toole et al., 1989). O'Toole et al. (1989) highlighted that the majority of triathletes with back pain also had another injury present at the same time.

In wider triathlon literature, cycling is 
uggested to be a major risk factor for back pain; Manninen and Kallinen (1996) concluded the majority self-resolved within seven days and in $19 \%$ it lasted over three months, suggesting disc pathology. The ankle and foot were also a frequently cited area of overuse injury (Egermann et al., 2003; O'Toole et al., 1989) (up to 61\%). Diagnoses included Achilles tendinitis, shin splints, tendinitis of the ankle, plantar fasciitis and tendinitis of the foot O'Toole et al. (1989). Achilles tendon injuries specifically, occurred in up to $27 \%$ and were more frequent in elite athletes (Egermann et al., 2003). Although Vleck et al. (2010) reported a significant number of Achilles tendon injuries in elite Ironman athletes, it was more frequent in elite Olympic distance triathletes.

Running has been previously reported as the most frequent phase within triathlon to attribute injury to (58\%-72\%) followed by cycling and then swimming (Burns et al., 2003; Clements et al., 1999; Collins et al., 1989; Korkia et al., 1994; Massimino et al., 1988; Vleck and Garbutt 1998). In this review, only two studies associated injuries with the phase of triathlon (swimming, cycling, running) (Egermann et al., 2003; Vleck et al., 2010). Both studies had a higher association of injuries during both running and cycling (statistical significance for all analyses was set at 0.05 in both studies), however, running was associated with more overuse injuries and cycling with acute injuries (Egermann et al., 2003; Vleck et al., 2010). Additionally, Andersen et al. (2013) was able to determine the overall percentage of time triathletes spent training in each discipline over a 26 week period: $48 \%$ cycling, $24 \%$ running, $12 \%$ swimming and $16 \%$ on other activities, such as weightlifting. Swimming had the lowest incidence of musculoskeletal injuries associated in the literature for the whole of triathlon, ranging from 5 to $11 \%$ (Collins et al., 1989; Korkia et al., 1994; Massimino et al., 1988). However, it has been noted as having the greatest potential for catastrophe, namely mortality (USA Triathlon Fatality Incidents Study - Team USA, 2012). In this systematic review, the study of Egermann et al. (2003) was the only one which included an acute injury incidence for swimming (5.8\%). Vleck et al. (2010) included the overuse injury incidence in swimming (16\%). Shoulder injuries were the most common type of swimming injury in triathlon
(Bales and Bales, 2012), with a highest reported incidence of $42 \%$ Andersen et al. (2013). Musculoskeletal injuries in swimming were rarer than in cycling and running.

Not only is more time spent during competition compared with other triathlon events, but more time is also spent during training. Egermann et al. (2003) reported a positive correlation between the number of training hours and injury incidence. Lower injury rates were noted in elite athletes (Hiller et al., 1987; Vleck et al., 2010) suggesting that the quality of training may be influential.

The main limitation of this research is the small number of studies reporting on injuries sustained during long-distance triathlon training and competition. The overall quality of the published evidence is low. No single study answered all the questions or applied gold standard sports injury surveillance methodology, such as prospectively designed studies with injury assessment by experienced clinicians. Only two studies were prospective (Andersen et al., 2013; Rimmer and Coniglione, 2012), the remainder were all retrospective. Two studies required recall of the injuries by the athletes themselves (Egermann et al., 2003; Vleck et al., 2010). Clinicians were involved in only two studies (Hiller et al., 1987; Rimmer and Coniglione, 2012). It had been decided not to include case reports in the analysis as this would have introduced bias in the reporting of the most common injuries sustained by long-distance triathletes.

\section{Conclusions}

Overuse injury was the most frequently reported musculoskeletal injury by long-distance triathletes in this review. Acute injuries were less frequent. Elite long-distance triathletes may have a lower incidence of both overuse and acute injuries and amateurs an increased rate of acute injuries. The knee was the most common anatomical location affected, followed by lower back and ankle and foot injuries. Running and cycling were the most frequently cited disciplines to cause injuries, the former leading to overuse injuries and the latter to acute ones. 


\section{References}

A journey like no other - Xtri World Tour. (n.d.). Retrieved 1 August 2020, from https://xtriworldtour.com/

Andersen, C. A., Clarsen, B., Johansen, T. V., \& Engebretsen, L. (2013). High prevalence of overuse injury among iron-distance triathletes. British Journal of Sports Medicine, 47(13), 857-861. https://doi.org/10.1136/bjsports-2013-092397

Bales, J., \& Bales, K. (2012). Swimming overuse injuries associated with triathlon training. Sports Medicine and Arthroscopy Review, 20(4), 196-199. https://doi.org/10.1097/JSA.0b013e318261093b

Burns, J., Keenan, A.-M., \& Redmond, A. C. (2003). Factors Associated With Triathlon-Related Overuse Injuries. Journal of Orthopaedic \& Sports Physical Therapy, 33(4), 177-184. https://doi.org/10.2519/jospt.2003.33.4.177

Caine, D. J., Harmer, P. A., \& Schiff, M. A. (2010). Epidemiology Of Injury In Olympic Sports: Vol. Volume XVI Of The Encyclopedia Of Sports Medicine.

https://stillmed.olympic.org/media/Document\%20Library/OlympicOrg/IOC/Who-WeAre/Commissions/Medical-and-Scientific-Commission/Encyclopaedia/2010_Caine.pdf

Clarsen, B., Myklebust, G., \& Bahr, R. (2013). Development and validation of a new method for the registration of overuse injuries in sports injury epidemiology: The Oslo Sports Trauma Research Centre (OSTRC) Overuse Injury Questionnaire. British Journal of Sports Medicine, 47(8), 495-502. https://doi.org/10.1136/bjsports-2012-091524

Clements, K., Yates, B., \& Curran, M. (1999). The prevalence of chronic knee injury in triathletes. British Journal of Sports Medicine, 33(3), 214-216.

Collins, K., Wagner, M., Peterson, K., \& Storey, M. (1989). Overuse injuries in triathletes. A study of the 1986 Seafair Triathlon. The American Journal of Sports Medicine, 17(5), 675-680. https://doi.org/10.1177/036354658901700515

Egermann, M., Brocai, D., Lill, C. A., \& Schmitt, H. (2003). Analysis of injuries in long-distance triathletes. International Journal of Sports Medicine, 24(4), 271-276. https://doi.org/10.1055/s-2003-39498

Gosling, C. M., Forbes, A. B., \& Gabbe, B. J. (2013). Health professionals' perceptions of musculoskeletal injury and injury risk factors in Australian triathletes: A factor analysis. Physical Therapy in Sport: Official Journal of the Association of Chartered Physiotherapists in Sports Medicine, 14(4), 207-212. https://doi.org/10.1016/j.ptsp.2012.09.004

Gosling, C. M., Gabbe, B. J., \& Forbes, A. B. (2008). Triathlon related musculoskeletal injuries: The status of injury prevention knowledge. Journal of Science and Medicine in Sport, 11(4), 396-406. https://doi.org/10.1016/j.jsams.2007.07.009

Hiller, W. D. B., O'Toole, M. L., Fortess, E. E., Laird, R. H., Imbert, P. C., \& Sisk, T. D. (1987). Medical and physiological considerations in triathlons. The American Journal of Sports Medicine, 15(2), 164-167. https://doi.org/10.1177/036354658701500212

Ireland, ML, Micheli, L.J. (1987). Triathletes: Biographic data, training, and injury patterns. Ann. Sports Med., 3, 117-120.

IRONMAN. (n.d.). Retrieved 1 August 2020, from https://www.ironman.com/

Kienstra, C. M., Asken, T. R., Garcia, J. D., Lara, V., \& Best, T. M. (2017). Triathlon Injuries: Transitioning from Prevalence to Prediction and Prevention. Current Sports Medicine Reports, 16(6), 397-403. https://doi.org/10.1249/JSR.0000000000000417

Korkia, P. K., Tunstall-Pedoe, D. S., \& Maffulli, N. (1994). An epidemiological investigation of training and injury patterns in British triathletes. British Journal of Sports Medicine, 28(3), 191-196. https://doi.org/10.1136/bjsm.28.3.191

Manninen, J. S., \& Kallinen, M. (1996). Low back pain and other overuse injuries in a group of Japanese triathletes. British Journal of Sports Medicine, 30(2), 134-139. https://doi.org/10.1136/bjsm.30.2.134

Massimino, FA \& Armstrong, MA. (1988). Common triathlon injuries: Special consideration for multisport training.

McHardy, A., Pollard, H., \& Fernandez, M. (2006). Triathlon injuries: A review of the literature and discussion of potential injury mechanisms. Clinical Chiropractic, 9(3), 129-138. https://doi.org/10.1016/j.clch.2006.04.001 
O’Toole, M. L., Hiller, W. D., Smith, R. A., \& Sisk, T. D. (1989). Overuse injuries in ultraendurance triathletes. The American Journal of Sports Medicine, 17(4), 514-518. https://doi.org/10.1177/036354658901700411

Rimmer, T., \& Coniglione, T. (2012). A temporal model for nonelite triathlon race injuries. Clinical Journal of Sport Medicine: Official Journal of the Canadian Academy of Sport Medicine, 22(3), 249-253. https://doi.org/10.1097/JSM.0b013e318249945b

Shaw, T., Howat, P., Trainor, M., \& Maycock, B. (2004). Training patterns and sports injuries in triathletes. Journal of Science and Medicine in Sport, 7(4), 446-450. https://doi.org/10.1016/s1440-2440(04)80262-7

Statistics. (n.d.). Retrieved 5 February 2021, from https://www.britishtriathlon.org/media/statistics

Ultraman World Championships. (n.d.). Retrieved 1 August 2020, from http://ultramanlive.com/

USA Triathlon Fatality Incidents Study-Team USA. (2012, October 1). USA Triathlon. file:///Users/jh/Downloads/USATFinalReport_24OCT12.pdf

Vleck, V. E., \& Garbutt, G. (1998). Injury and training characteristics of male Elite, Development Squad, and Club triathletes. International Journal of Sports Medicine, 19(1), 38-42. https://doi.org/10.1055/s-2007971877

Vleck, V., Millet, G., \& Alves, F. (2013). Triathlon Injury-An update. Schweizerische Zeitschrift Fur Sportmedizin Und Sporttraumatologie, 61, 10-16.

Vleck, VE. (2003). Triathlete training and injury analysis [Ph.D.].

Vleck, Veronica E., Bentley, D. J., Millet, G. P., \& Cochrane, T. (2010). Triathlon event distance specialization: Training and injury effects. Journal of Strength and Conditioning Research, 24(1), 30-36. https://doi.org/10.1519/JSC.0b013e3181bd4cc8

Wilk, B. R., Fisher, K. L., \& Rangelli, D. (1995). The Incidence of Musculoskeletal Injuries in an Amateur Triathlete Racing Club. Journal of Orthopaedic \& Sports Physical Therapy, 22(3), 108-112. https://doi.org/10.2519/jospt.1995.22.3.108

\section{Corresponding author:}

John-Henry Rhind,

Robert Jones Agnes Hunt Hospital

Tel. 07899998882

E-mail: rhind99@hotmail.com 